\title{
Effectiveness of Internet- and mobile-based psychological interventions for the prevention of mental disorders: a systematic review and meta-analysis protocol
}

\author{
Lasse Sander $^{1,2^{*}}$, Leonie Rausch ${ }^{1}$ and Harald Baumeister ${ }^{3}$
}

\begin{abstract}
Background: Despite progress in developing technologies for health promotion and disease treatment, mental disorders remain highly prevalent. In light of the associated personal and societal burden, primary prevention of new onset disorder can be seen as a primary challenge for health care systems and society. Internet- and mobile-based interventions (IMIs) are a promising approach to scale up preventive measures to a population level. The present protocol describes the rationale and design of a systematic review on the effectiveness of IMIs for the prevention of mental disorders.

Methods/design: A systematic literature search (MEDLINE, PsycINFO, CENTRAL) will be conducted. Keywords will target the topics "prevention", "mental disorders", and "Internet and mobile-based interventions". Studies eligible for inclusion will be retrieved, and data will be extracted and evaluated (design, population, outcomes, sample size, duration of intervention and follow-up, drop-out rate) by two independent researchers. Quality of evidence will be assessed, and results will be synthesized qualitatively and pooled meta-analytically when outcome data are comparable in terms of endpoints, assessments, and target disorders.
\end{abstract}

Discussion: Prevention of mental disorders is one of the major emerging global health challenges. This review and meta-analysis will contribute to our understanding of the potential role for IMIs to help address this challenge.

Systematic review registration: PROSPERO CRD42015026781

Keywords: Prevention, Systematic review, Mental disorders, Internet and mobile-based, Online

\section{Background}

Mental disorders are highly prevalent [1] and associated with a high disease burden for affected individuals and society [2-4]. Wittchen and colleagues reported a 1-year prevalence rate of mental disorders (27 diagnoses included) in the EU population of $38.2 \%$. Mental disorders are prevalent across age groups, with anxiety disorders

\footnotetext{
* Correspondence: lasse.sander@psychologie.uni-freiburg.de

'Department of Rehabilitation Psychology and Psychotherapy, Institute of Psychology, University of Freiburg, Engelbergerstr. 41, Freiburg im Breisgau D-79085, Germany

2Department of Medical Psychology and Medical Sociology, Faculty of Medicine University of Freiburg, Hebelstr. 29, Freiburg im Breisgau D-79104, Germany Full list of author information is available at the end of the article
}

(14.0\%), insomnia (7.0 \%), and major depression (6.9\%) being the most frequent disorders [1]. Compared to 2005 data, these authors note that neither a substantial decrease in the prevalence nor improvements in care or treatment were found, and less than a third of people affected received any treatment [5]. Thus, despite increasing effectiveness levels of available treatments and utilizing them more widely, interventions to reduce the rate of onset of mental disorder must also be developed and implemented into health care systems. Experts agree that prevention of mental disorders, when possible, reduces burden most significantly [6]. Efforts to support the development of effective preventive interventions are urgently needed. 
Recent reviews and meta-analyses highlight that psychological preventive interventions are able to substantially reduce rates of new onset of different types of mental disorders [7-10]. Not surprisingly, studies examining the effects of preventive measures aimed at populations carrying potential risk indicators (=selective prevention) or populations with prodromal syndromes or biological markers to mental disorders (=indicated prevention) are more common than studies geared toward the general population, regardless of risk status (=universal prevention) [7].

Using the Internet as the medium for the delivery of psychological prevention interventions has several advantages compared to traditional intervention settings. Internet- and mobile-based interventions (IMIs) are easy to access, enabling users to flexibly integrate interventions into their daily life without local or temporal boundaries [11]. Moreover, users can set the pace for working with these interventions and can go through each session as frequently or as quickly as they like. For users fearing stigmatization, the anonymity offered by IMIs can be appealing. First studies also found Internetbased interventions to be cost-effective [12-14], a crucial element in light of limited resources in many health care systems.

Additionally, access to and use of the Internet have grown rapidly over the last decade across the globe [15]. Mental health professionals could leverage this trend of increasing Internet use, which often includes getting health information and support [16].

Several studies have examined the effectiveness of Internet-based prevention interventions for specific mental disorders (e.g., $[17,18])$. Prior reviews and metaanalyses have been published focusing on IMIs for the prevention of eating disorders [19-21] and substancerelated/addictive disorders [22, 23]. To our knowledge, there is no previous systematic literature review summarizing the existing research on Internet- and mobilebased prevention interventions for mental disorders in general. Therefore, the results of this review and metaanalysis will provide an overview of this field of research that can benefit clinicians, public health policy makers, and researchers. The present protocol describes the rationale and design of the systematic review and metaanalysis.

\section{Methods/design}

This review is registered with PROSPERO (registration CRD42015026781).

\section{Aims}

The review aims to synthesize the evidence base of randomized controlled trials (RCTs) reporting the effectiveness of Internet- and mobile-based prevention interventions of mental disorders. The review will be reported according to the Preferred Reporting Items for Systematic Reviews and Meta-Analyses (PRISMA) guidelines [24].

\section{Search strategy}

Relevant articles will be identified by searching electronic databases. Databases included are Cochrane Central Register of Controlled trials (CENTRAL), PsycINFO, and MEDLINE. A sensitive search strategy will be used (see Additional file 1). The search will be complemented by a review of reference lists from identified publications and a search of the WHO International Clinical Trials Registry Platform (ICTRP) to include ongoing trials. When indicated, study authors will be contacted to obtain further information in order to clarify study characteristics. When study protocols are identified without subsequent publication of results, authors will be contacted to obtain missing or unpublished data and determine eligibility for inclusion in this review.

\section{Eligibility criteria}

Population: Studies are eligible for inclusion if they (a) focus on an adult target population and (b) include adults without a diagnosis of the target mental disorder at baseline (primary prevention intervention). (c) Mental disorder should be assessed by means of standardized interviews (e.g., SCID, CIDI), validated self-reports (e.g., BDI), clinician-rated scales (e.g., HAMD) with normed cut-off points, or diagnosis by health care professionals. Studies on the prevention of substance-related/addictive disorders will be excluded, as this represents a frequently studied and already elsewhere reviewed specific subgroup of prevention research $[22,23]$ and these interventions typically follow an educational or health behavior change model rather than a psychotherapeutic intervention model (see (d)).

Intervention: (d) Interventions must be based on psychological interventions. The definition of "psychological intervention" is taken from Kampling and colleagues [25] and refers to cognitive behavioral therapy, psychodynamic psychotherapy, behavior therapy or behavior modification, systemic therapy, third wave cognitive behavioral therapies, humanistic therapies, integrative therapies, and other psychological-oriented interventions. (e) Interventions must be provided in an online setting, defined as online-, Internet-, Web-, or mobile-based. Interventions may vary concerning the amount of external guidance provided to participants. Self-help interventions will also be included. We will exclude studies on the relapse prevention of mental disorder, as these treatment maintenance interventions differ substantially from preventive interventions focused on the first or recurrent onset of mental disorders [25]. 
Comparison: (f) Studies must include a control group. This can be (enhanced) usual care, wait-list control group, another intervention, or no treatment.

Outcomes: (g) Studies examining onset will be considered, defined as the percentage of persons who developed the mental disorder under study from pre- to follow-up assessment. In addition to the data from standardized clinical interviews (e.g., SCID-IV [26]), we will include studies reporting only reporting symptom severity scores, when validated rating scales with normed cutoff points (referencing onset of disorder or diagnosis) have been used. To be able to meaningfully comment on any post-intervention reduction of incidence, studies must (h) include a follow-up assessment at 3 months or longer post-randomization.

Study type: (i) only RCTs that are available in full text will be eligible for this review. Studies must be published in English or German.

\section{Study selection process}

The selection of articles will be conducted by two independent reviewers (LS, LR). In the first step, they will screen all titles and abstracts yielded by the database search. In the second step, full texts of the selected articles will be retrieved and screened in terms of the aforementioned eligibility criteria. Reference lists of finally included articles will be screened in the same way. Disagreement will be resolved by a discussion among the reviewers. When needed to resolve disagreement, a third reviewer (HB) will be consulted. To illustrate the study selection process and reasons for exclusion, a PRISMA flow chart [24] will be provided (see Fig. 1).

\section{Data extraction}

The following data items will be extracted for each study, when available: (a) study identification items (first

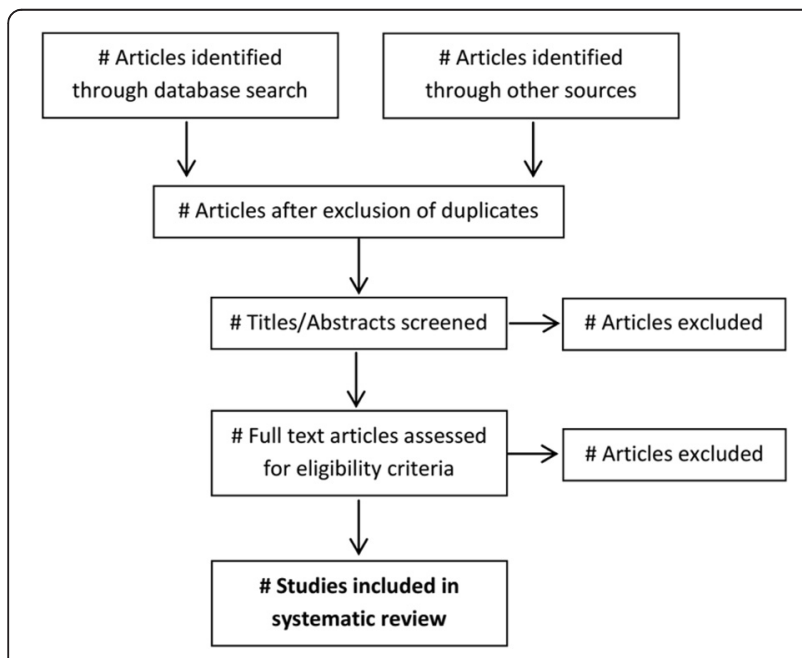

Fig. 1 Study selection process (adapted from [24]) author, year of publication), (b) study design characteristics (sample size, intervention design/type, level of human support/guidance target mental disorder, control group, type of assessments, duration of intervention, length of follow-up assessments), (c) target population items (age, gender), (d) setting (nationality, environment, recruitment strategy), (e) drop-out rate and (f) clinical outcomes (onset and/or severity of disorder). If outcomes are assessed by several instruments, data will be extracted as follows: (1) Onset data from structured interviews will be prioritized. (2) Data from clinician-rated scales will be prioritized over self-report questionnaires. (3) Self-report questionnaires will be prioritized over diagnosis by health care professionals. (4) In case where several outcome measures of the same hierarchy level are used in one study, we will select the outcome measure that is used most frequently across eligible studies or else randomly select one outcome measure. To avoid bias, a second reviewer will check the extracted data.

\section{Quality assessment}

In order to evaluate the quality of research, two independent reviewers will assess the risk of bias using the Cochrane Collaboration's tool for assessing risk of bias in RCTs [27]. As recommended, each study will be assessed for procedures in the following domains: (a) random sequence generation, (b) allocation concealment, (c) blinding of participants, personnel, and outcome assessors, (d) incomplete outcome data (drop-out, intention-to-treat), (e) selective outcome reporting, and (f) other threats to validity. Studies will be rated as showing a "low" or "high" risk of bias according to the aforementioned criteria. Of note, in the implementation of psychological interventions, blinding of health care providers (if a guided intervention was provided) or patients concerning the treatment is not possible. This results in a "high" risk of bias rating on this criterion. However, outcome assessors can remain unaware of the treatment allocation of patients.

\section{Data synthesis and presentation}

A detailed description of the results for all included studies will be provided in text and tables. Characteristics of selected studies will be listed and qualitatively described. Characteristics include study design and characteristics (sample size, duration, follow-up period), patient population (age, gender), intervention characteristics (name, intervention content (e.g., CBT), technical implementation (e.g., Web-only or Web- and mobilebased), duration, level of human support/guidance), study and intervention drop-out rate, assessment tool used to determine presence of mental disorder (clinical interview, questionnaire), type of prevention (universal, selective, indicative), recruitment procedure, target 
disorder to be prevented, and any covariates assessed (list of variables).

\section{Meta-analysis}

Data analysis will be performed using Review Manager 5.3 software from the Cochrane Collaboration. Metaanalytic pooling will be conducted when at least three studies report outcome parameters on the same specific mental disorder (i.e., specific mental disorder according to DSM/ICD such as major depression or social phobia assessed as described in "eligibility criteria: population: (c)"). Only studies showing less than substantial statistical heterogeneity will be pooled. Heterogeneity will be evaluated with the $I^{2}$ statistic. An $I^{2}$ of $0-60 \%$ can be regarded as not important to moderate, while $I^{2}>60 \%$ indicates substantial heterogeneity [27]. Focusing on specific mental disorders rather than pooling results across disorder entities will limit clinical heterogeneity of pooled estimates. The random-effects method using the inverse-variance model for pooled estimates on the prevention of mental disorders (hazard ratios, odds ratios with $95 \% \mathrm{CI}$ ) and for the pooled standardized mean difference (SMD with $95 \% \mathrm{CI}$ ) of the severity level of the respective mental disorder will be used. Outcome assessment data (i.e., on the onset and the severity differences between trial arms) will be pooled, subdivided for short-term (post-treatment assessment), medium-term ( $\leq 6$ months post-randomization follow-up), and longterm ( $>6$ months post-randomization follow-up) trial effects. For further comparisons concerning content and form of intervention, subgroup analysis will be performed if feasible.

\section{Discussion}

This systematic review and meta-analysis will add to the previous research by summarizing, synthesizing, and discussing the existing literature on Internet- and mobilebased prevention interventions for mental disorders. The findings of this review will extend beyond previous systematic reviews in this field (e.g., [20, 28]), as results will extend to a more comprehensive range of mental disorders. Thus, the proposed systematic review and meta-analysis will provide a valuable overview and synthesis of the entire field of Internet- and mobile-based mental disorder prevention interventions for clinical practitioners, researchers, and public health policy makers.

The following characteristics will contextualize the findings and generalizability of this review. In service of providing a broad review on the broad scope of mental disorders, this review will include trials that might differ substantially regarding their target population, methods, interventions, control groups, assessments, and outcomes. While this will provide a comprehensive overview on the prevention of mental disorders by means of
Internet- and mobile-based interventions, heterogeneity in clinical, methodological, and statistical approaches will necessarily limit the amount of quantitative pooling of studies. Moreover, the language bias (including only studies published in English or German) may lead to an overestimation of effects, as statistically significant results are more likely to be published in the English language [29]. The publication bias of only significant findings being published may also contribute to the limitation of results [30]. To address this issue, we will attempt to include unpublished and non-significant studies by contacting principal investigators of studies and study protocols.

In the past, efforts toward prevention of mental disorders were often limited due to limited resources of health care systems as well as challenges inherent in providing an intervention en masse [31]. Using the Internet as the medium for provision has the potential to overcome this limitation. To guide public health policy, the level of effectiveness of an intervention is a crucial information for decisions on the implementation of new health care approaches. An important issue concerning Internet- and mobile-based interventions is attrition [32-34]. We will therefore list and discuss treatment and intervention drop-out rates in this review.

The proposed review will go beyond summarizing the existing evidence by also qualitatively analyzing the past and ongoing interventions. In this way, gaps in the previous research will be addressed, essential characteristics of prevention interventions will be outlined, and missing subfields in research will be identified.

Given the numerous upcoming Internet- and mobilebased prevention intervention trials on mental disorders and the absence of a respective systematic review, the proposed review is urgently needed and will substantially add to the current evidence. Our findings will help researchers to identify options for future directions and public health policy makers to estimate the potential of mental health prevention interventions.

\section{Additional file}

Additional file 1: Table showing the search strings for CENTRAL, MEDLINE, and PsycINFO.

\section{Abbreviations \\ CENTRAL: Cochrane Central Register of Controlled Trials; ICTRP: International Clinical Trials Registry Platform; IMI: Internet and mobile-based intervention; PRISMA: Preferred Reporting Items for Systematic Reviews and Meta-Analyses; $\mathrm{RCT}$ : randomized controlled trial; WHO: World Health Organization.}

Competing interests

The authors declare that they have no competing interests. 


\section{Authors' contributions}

$L S, L R$, and $H B$ were involved in the concept and review design of the study and data analysis plan. All authors had major contributions to the write-up and editing of the manuscript and read and approved the final manuscript.

\section{Acknowledgements}

We would like to thank Yannik Terhorst for proofreading of the manuscript and Mary Wyman for language editing. The article processing charge was funded by the German Research Foundation (DFG) and the Albert Ludwigs University Freiburg in the funding program Open Access Publishing. LS is supported by the Federal Ministry of Education and Research, Germany (project: Effectiveness of a guided Web-based intervention for depression in back pain rehabilitation aftercare, grant number: 01GY1330A). The funders had no role in the study design, data collection and analysis, decision to publish, or preparation of the manuscript.

\section{Author details}

'Department of Rehabilitation Psychology and Psychotherapy, Institute of Psychology, University of Freiburg, Engelbergerstr. 41, Freiburg im Breisgau D-79085, Germany. ${ }^{2}$ Department of Medical Psychology and Medical Sociology, Faculty of Medicine, University of Freiburg, Hebelstr. 29, Freiburg im Breisgau D-79104, Germany. ${ }^{3}$ Department of Clinical Psychology and Psychotherapy, Institute of Psychology and Education, University of Ulm, Albert-Einstein-Allee 47, Ulm D-89081, Germany

Received: 18 December 2015 Accepted: 8 February 2016

Published online: 16 February 2016

\section{References}

1. Wittchen H-U, Jacobi F, Rehm J, Gustavsson A, Svensson M, Jönsson B, et al. The size and burden of mental disorders and other disorders of the brain in Europe 2010. Eur Neuropsychopharmacol. 2011;21(9):655-79.

2. Gustavsson A, Svensson M, Jacobi F, Allgulander C, Alonso J, Beghi E, et al. Cost of disorders of the brain in Europe 2010. Eur Neuropsychopharmacol. 2011:21(10):718-79.

3. World Health Organization. The global burden of disease: 2004 update. Geneva: WHO Press; 2008. http://www.who.int/healthinfo/global_burden_ disease/GBD_report_2004update_full.pdf. Accessed Sep 52015.

4. World Health Organization. The European mental health action plan. Çeşme Izmir, Turkey; 2013. http://apps.who.int/iris/bitstream/10665/89966/1/ 9789241506021_eng.pdf?ua=1. Accessed Oct 52015.

5. Wittchen HU, Jacobi F. Size and burden of mental disorders in Europe-a critical review and appraisal of 27 studies. Eur Neuropsychopharmacol. 2005:15(4):357-76

6. Jacka FN, Reavley NJ. Prevention of mental disorders: evidence, challenges and opportunities. BMC Med. 2014;12(1):75.

7. Van Zoonen K, Buntrock C, Ebert DD, Smit F, Reynolds CF, Beekman ATF, et al. Preventing the onset of major depressive disorder: a meta-analytic review of psychological interventions. Int J Epidemiol. 2014;43(2):318-29.

8. Stice $\mathrm{E}$, Shaw $\mathrm{H}$, Marti $\mathrm{CN}$. A meta-analytic review of eating disorder prevention programs: encouraging findings. Annu Rev Clin Psychol. 2007;3(1):207-31.

9. Cuijpers P, Van Straten A, Smit F. Preventing the incidence of new cases of mental disorders: a meta-analytic review. J Nerv Ment Dis. 2005;193(2):119-25

10. Cuijpers P. Prevention: an achievable goal in personalized medicine Dialogues Clin Neurosci. 2009;11(4):447-54.

11. Cuijpers P, Van Straten A, Warmerdam L, Van Rooy MJ. Recruiting participants for interventions to prevent the onset of depressive disorders: possible ways to increase participation rates. BMC Health Serv Res. 2010:10(1):181-7.

12. Donker T, Blankers M, Hedman E, Ljótsson B, Petrie K, Christensen H. Economic evaluations of internet interventions for mental health: a systematic review. Psychol Med. 2015;45(16):3357-76.

13. Titov N, Dear BF, Ali S, Zou JB, Lorian CN, Johnston L, et al. Clinical and costeffectiveness of therapist-guided internet-delivered cognitive behavior therapy for older adults with symptoms of depression: a randomized controlled trial. Behav Ther. 2015:46(2):193-205.

14. Warmerdam L, Smit F, van Straten A, Riper H, Cuijpers P. Cost-utility and cost-effectiveness of internet-based treatment for adults with depressive symptoms: randomized trial. J Med Internet Res. 2010;12(5):e53.

15. Internet Society. Global Internet Report. 2014. http://www.internetsociety.org/ sites/default/files/Global_Internet_Report_2014.pdf. Accessed Oct 152015.
16. Horgan A, Sweeney J. Young students' use of the internet for mental health information and support. J Psychiatr Ment Health Nurs. 2010;17(2):117-23.

17. Imamura K, Kawakami N, Furukawa TA, Matsuyama Y, Shimazu A, Umanodan $\mathrm{R}$, et al. Does internet-based cognitive behavioral therapy (iCBT) prevent major depressive episode for workers? A 12-month follow-up of a randomized controlled trial. Psychol Med. 2015:54(9):1907-17.

18. Jacobi C, Morris L, Beckers C, Bronisch-Holtze J, Winter J, Winzelberg AJ, et al. Maintenance of internet-based prevention: a randomized controlled trial. Int J Eat Disord. 2007:40(2):114-9.

19. Schlegl S, Bürger C, Schmidt L, Herbst N, Voderholzer U. The potential of technology-based psychological interventions for anorexia and bulimia nervosa: a systematic review and recommendations for future research. J Med Internet Res. 2015;17(3), e85.

20. Beintner I, Jacobi C, Taylor CB. Effects of an internet-based prevention programme for eating disorders in the USA and Germany-a meta-analytic review. Eur Eat Disord Rev. 2012;20(1):1-8.

21. Dölemeyer R, Tietjen A, Kersting A, Wagner B. Internet-based interventions for eating disorders in adults: a systematic review. BMC Psychiatry. 2013;13:207.

22. Champion KE, Newton NC, Barrett EL, Teesson M. A systematic review of school-based alcohol and other drug prevention programs facilitated by computers or the internet. Drug Alcohol Rev. 2013;32(2):115-23.

23. Tait RJ, Spijkerman R, Riper H. Internet and computer based interventions for cannabis use: a meta-analysis. Drug Alcohol Depend. 2013;133(2):295-304.

24. Liberati A, Altman DG, Tetzlaff J, Mulrow C, loannidis JP, Clarke M, et al. The PRISMA statement for reporting systematic reviews and meta-analyses of studies that evaluate health care interventions: explanation and elaboration. Ann Intern Med. 2009;151(4):W65-94.

25. Kampling $\mathrm{H}$, Baumeister $\mathrm{H}$, Jäckel Wilfried $\mathrm{H}$, Mittag O. Prevention of depression in chronically physically ill adults. Cochrane Database Syst Rev. 2014;8.

26. First MB, Spitzer RL, Gibbon M, Williams JBW. The structured clinical interview for DSM-IV axis I disorders (SCID-I) and the structured clinical interview for DSM-IV axis II disorders (SCID-II). Washington, DC: American Psychiatric Press; 2002.

27. Higgins JPT, Green S, editors. Cochrane handbook for systematic reviews of interventions. Chichester: John Wiley \& Sons; 2008.

28. Riper H, Blankers M, Hadiwijaya H, Cunningham J, Clarke S, Wiers R, et al. Effectiveness of guided and unguided low-intensity internet interventions for adult alcohol misuse: a meta-analysis. PLoS One. 2014;9(6), e99912.

29. Egger M, Zellweger-Zähner T, Schneider M, Junker C, Lengeler C, Antes G. Language bias in randomised controlled trials published in English and German. Lancet. 1997;350(9074):326-9.

30. Ioannidis JPA. Why most published research findings are false. PLoS Med. 2005;2(8), e124.

31. Zechmeister I, Kilian R, McDaid D. Is it worth investing in mental health promotion and prevention of mental illness? A systematic review of the evidence from economic evaluations. BMC Public Health. 2008:8(1):20.

32. Christensen $H$, Griffiths KM, Farrer L. Adherence in internet interventions for anxiety and depression: systematic review. J Med Internet Res. 2009;11(2), e13.

33. Karyotaki E, Kleiboer A, Smit F, Turner DT, Pastor AM, Andersson G, et al. Predictors of treatment dropout in self-guided web-based interventions for depression: an "individual patient data" meta-analysis. Psychol Med. 2015;1-10.

34. Melville KM, Casey LM, Kavanagh DJ. Dropout from internet-based treatment for psychological disorders. Br J Clin Psychol. 2010;49(4):455-71.

\section{Submit your next manuscript to BioMed Central and we will help you at every step:}

- We accept pre-submission inquiries

- Our selector tool helps you to find the most relevant journal

- We provide round the clock customer support

- Convenient online submission

- Thorough peer review

- Inclusion in PubMed and all major indexing services

- Maximum visibility for your research

Submit your manuscript at www.biomedcentral.com/submit 